\title{
What is the yield of malaria reactive case detection in the Greater Mekong Sub-region? A review of published data and meta-analysis
}

\author{
Jacqueline Deen $^{1 *}$ (D), Mavuto Mukaka ${ }^{2,3}$ and Lorenz von Seidlein ${ }^{2,3}$
}

\begin{abstract}
Background: Reactive malaria case detection involves the screening of those in contact with index cases and is used in countries in the Greater Mekong Sub-region. The yield of reactive case detection, defined here as the percentage of positive malaria cases among potential contacts who were screened, was assessed.

Methods: A literature search was conducted on PubMed to identify studies on reactive case detection in the Greater Mekong Sub-region. Eligible published articles were reviewed and pooled estimates from the studies were calculated, by type of malaria test used.

Results: Eighty-five publications were retrieved, of which 8 (9.4\%) eligible articles were included in the analysis. The yield from reactive case detection ranged from 0.1 to $4.2 \%$, with higher rates from PCR testing compared with microscopy and/or rapid diagnostic test. The overall yield from microscopy and/or rapid diagnostic test was $0.56 \%(95 \% \mathrm{Cl}$ $0.31-0.88 \%$ ), while that from PCR was $2.35 \%$ (95\% Cl 1.19-3.87\%). The two studies comparing different target groups showed higher yield from co-workers/co-travellers, compared with household contacts.

Conclusion: In low malaria transmission settings, the effectiveness of reactive case detection is diminishing. In the Greater Mekong Sub-region, modifying reactive case detection from household contacts to co-workers/co-travellers and from testing to presumptive treatment of targeted contacts, could increase the impact of this approach.
\end{abstract}

Keywords: Malaria, Active case detection, Reactive case detection

\section{Background}

The countries in the Greater Mekong Sub-region (GMS) maintain health systems for the diagnosis, management, and reporting of malaria cases detected by passive case detection (PCD). Early detection and appropriate treatment of patients who present to health workers is effective in the control of malaria. As malaria cases decline and regions like the GMS advance towards malaria elimination, more intensive methods than PCD are needed

\footnotetext{
*Correspondence: deen.jacqueline@gmail.com; jldeen@up.edu.ph ${ }^{1}$ Institute of Child Health and Human Development, National Institutes of Health, University of the Philippines, 623 P. Gil St, 1000 Manila, Philippines

Full list of author information is available at the end of the article
}

to accelerate the interruption of transmission and prevent reversals [1]. Active case detection (ACD) strategies require investigators to visit households, farms and forests and actively look for and treat malaria cases. ACD in the GMS is focused on Migrant Mobile Ethnic and Vulnerable (MMEV) populations, who are at increased risk because of limited access to health-care services [2]. ACD may take the form of reactive case detection (RACD) or proactive case detection (PACD), both of which have been adopted in the GMS countries. RACD involves the screening of those potentially in contact with malaria index cases, including their household members, other community members within a radius around the index case, co-workers and co-travellers. PACD involves

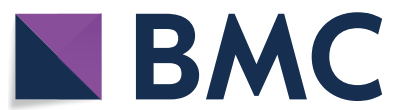

(c) The Author(s) 2021. This article is licensed under a Creative Commons Attribution 4.0 International License, which permits use, sharing, adaptation, distribution and reproduction in any medium or format, as long as you give appropriate credit to the original author(s) and the source, provide a link to the Creative Commons licence, and indicate if changes were made. The images or other third party material in this article are included in the article's Creative Commons licence, unless indicated otherwise in a credit line to the material. If material is not included in the article's Creative Commons licence and your intended use is not permitted by statutory regulation or exceeds the permitted use, you will need to obtain permission directly from the copyright holder. To view a copy of this licence, visit http://creativeco mmons.org/licenses/by/4.0/. The Creative Commons Public Domain Dedication waiver (http://creativecommons.org/publicdomain/ zero/1.0/) applies to the data made available in this article, unless otherwise stated in a credit line to the data. 
the general screening of high-risk populations before they present with malaria.

RACD is labour- and resource-intensive. The diagnoses of the index case needs to be confirmed and the contacts need to be traced, identified, informed, tested and treated, if found infected. Since speedy detection and treatment of infected contacts is of the essence, $a \leq 7$-day time window from diagnosis of the index case to the treatment of the contacts is recommended [3]. The yield of RACD, defined here as the number of secondary cases detected per number of contacts investigated, depends on the characteristics of the screened population, including occupation, mobility and the type of contact with the index case. The yield of RACD also varies by how the contacts are selected for screening following the identification of the index case, malaria transmission dynamics and endemicity of the area and the sensitivity of the diagnostic test used. Simulations using data from four villages on the Myanmar-Thailand border showed that RACD using rapid diagnostic tests (RDT) has a limited ability in halting transmission in regions of low and unstable transmission due to high spatial heterogeneity of cases, acquisition of malaria infections outside the village, as well as missing low density, asymptomatic infections, which can make up the majority of infections but cannot be detected with the available diagnostic tools [4]. These simulations may not reflect actual data or be generalizable to other areas of the GMS. To get a better understanding of the current, empirical yield of RACD the available published data on RACD in the GMS were reviewed.

\section{Methods}

PubMed was searched using the following search string: "(malaria) AND ("case detection" OR "1-3-7" OR "screen" and treat") AND ("Greater Mekong Subregion" OR Cambodia OR Laos OR Myanmar OR Thailand OR Vietnam OR Viet Nam OR China) AND ((“2010/01/01"[PDat]:"2020/09/15"[PDat]))", with no language restrictions. Titles and abstracts from the literature search were compiled in Endnote (Thomson Reuters, San Francisco, CA, USA).

Titles and abstracts were initially reviewed. Policy descriptions, commentaries and reviews; studies outside the GMS; qualitative and feasibility studies; and entomologic studies were excluded. The relevant full articles on ACD in the GMS were downloaded and reviewed in detail. Included studies were those wherein the screening for malaria started with a passively identified index case. Furthermore, the article had to report the number of contacts screened and the number who were positive for malaria by microscopy, RDT or PCR. The studies could be prospective or retrospective. Articles that did not start with passively detected index cases or did not report the number of contacts screened and the number who were malaria positive were excluded.

Information from each paper was extracted and entered into an Excel spread sheet (Microsoft ${ }^{\circledR}$ Office 2007, Seattle, WA, USA). The descriptive information included location, study period and characteristics of the index cases and contacts screened. The quantitative data collected included the number positive by microscopy, RDT and PCR.

The primary endpoint of this review is the yield of RACD, defined here as the percentage of positive malaria cases among the contacts who were screened, by type of malaria test performed (microscopy and/or RDT versus PCR). Forest plots of the percentage yields (with 95\% confidence intervals) from individual studies were created using Stata 16 (StataCorp, College Station, Texas, USA). The weights contributed by each study were calculated from random effects model directly from the Stata "metaprop" command [5]. $\mathrm{I}^{2}$ statistic was used to describe the percentage of variation across studies that is due to heterogeneity rather than chance.

The validity of this systematic review was established by adhering to the pre-defined inclusion and exclusion criteria (described above) to allow comparison across individual studies. We expect differences in field and laboratory methods techniques and included studies that met the basic inclusion and exclusion criteria. Publication bias was not systematically calculated since there is no standard for measuring the expected yield of RACD in the GMS.

\section{Results}

From a PubMed database search on 15 September 2020, 85 articles were retrieved (Fig. 1). Sixty-one (71.8\%) publications were excluded based on the review of the titles and abstracts. Twenty-four articles on ACD in the GMS were identified, downloaded and reviewed in detail. Of the $24,11(45.8 \%)$ articles that did not report the yield of reactive case investigation, four (16.7\%) that did not start with passively detected malaria index cases (these included mass blood surveys and PACD) and one (4.2\%) modelling study were excluded. Eight eligible articles [6-13] were included in the analysis, with one reporting on four sub-studies [13]. All eligible articles were in English, except for one (in Chinese) [7], which was translated prior to review.

The data from the eligible articles were summarized (Table 1). Seven $(63.6 \%)$ of the 11 study sites were in Cambodia, 3 (27.3\%) in China and one $(9.1 \%)$ in Thailand. The studies were conducted from 2011 to 2018 . The number of index cases ranged from one to 639 and the number of contacts screened ranged from 126 to 3,662. 
Search string resulted in 85 publications on PubMed, the titles and abstracts of which were reviewed

Excluded 61 publications:

- 24 malaria epidemiologic studies but not on reactive case detection

- 11 policy descriptions, commentaries and reviews

- 11 studies not in the GMS

- 8 qualitative studies

- 3 studies not on malaria

- 1 feasibility study

- 1 entomologic study

- 1 study protocol

- 1 erratum

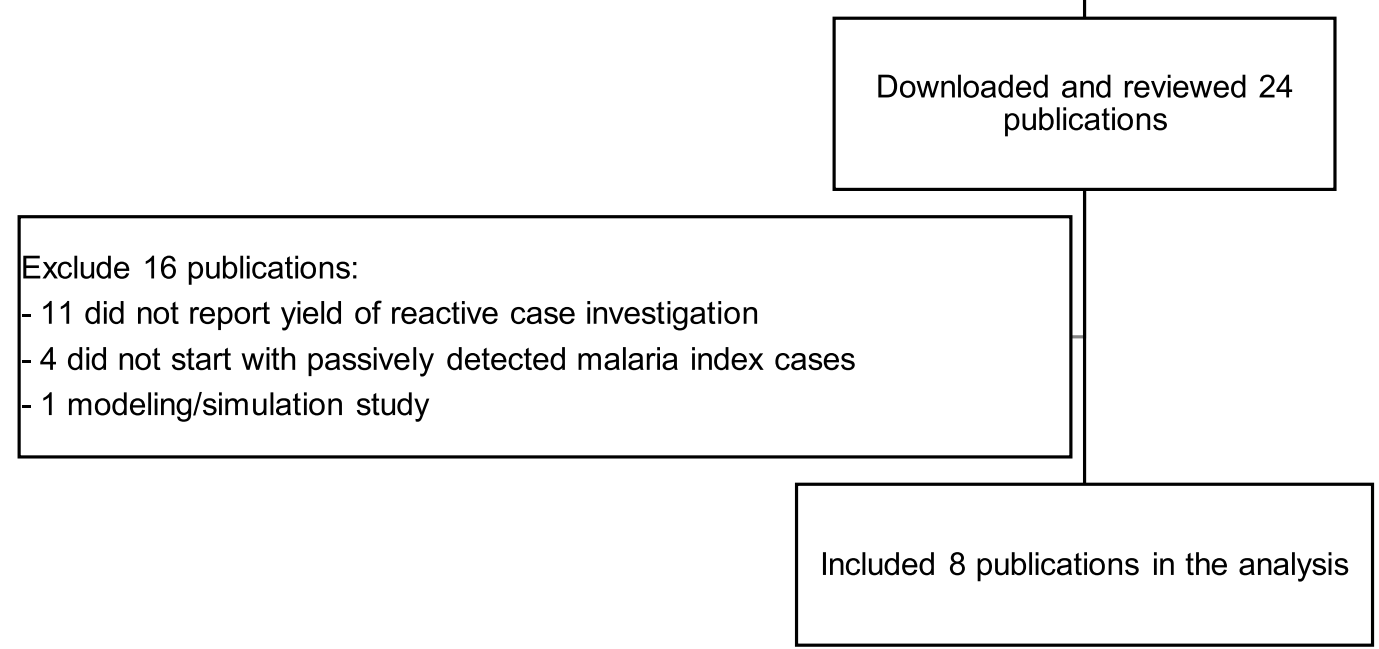

Fig. 1 Selection of publications included in the analysis

The yield from RACD ranged from 0.1 to $4.2 \%$, with higher rates from PCR testing compared with microscopy and/or RDT (Fig. 2a, b). The overall yield from microscopy and/or RDT was $0.56 \%$ (95\% CI $0.31-0.88 \%$ ), while that from PCR was $2.35 \%$ (95\% CI 1.19-3.87\%). There was a very high heterogeneity across studies using microscopy and/or RDT and PCR with an $\mathrm{I}^{2}$ statistic of 80.9 and $90.8 \%$, respectively. This suggests that 80.9 and $90.8 \%$ of the variation across studies is due to heterogeneity. The heterogeneity was statistically significant at $p<0.01$ for studies using microscopy and/or RDT and PCR. Two studies $[11,12]$ compared the yield of screening index cases' household members versus other contacts (co-workers and co-travellers) and found higher rates among the latter (Table 1 ).

\section{Discussion}

In this analysis of published studies on RACD in the GMS, we found an overall yield from microscopy and/ or RDT of roughly one case detected per 200 contacts tested. The yield from PCR was four times higher than microscopy and/or RDT but PCR is generally not used for screening because it is expensive, time- and labourintensive, and requires advanced laboratory capacity. Critically, the results from PCR testing of samples are not immediately available, delaying the treatment of infected contacts. To achieve the highest impact infected contacts should be diagnosed with an appropriate point of care test and treated immediately.

Standard PCR, as used in the studies reported here, had higher yield than RDT, but has been estimated to 


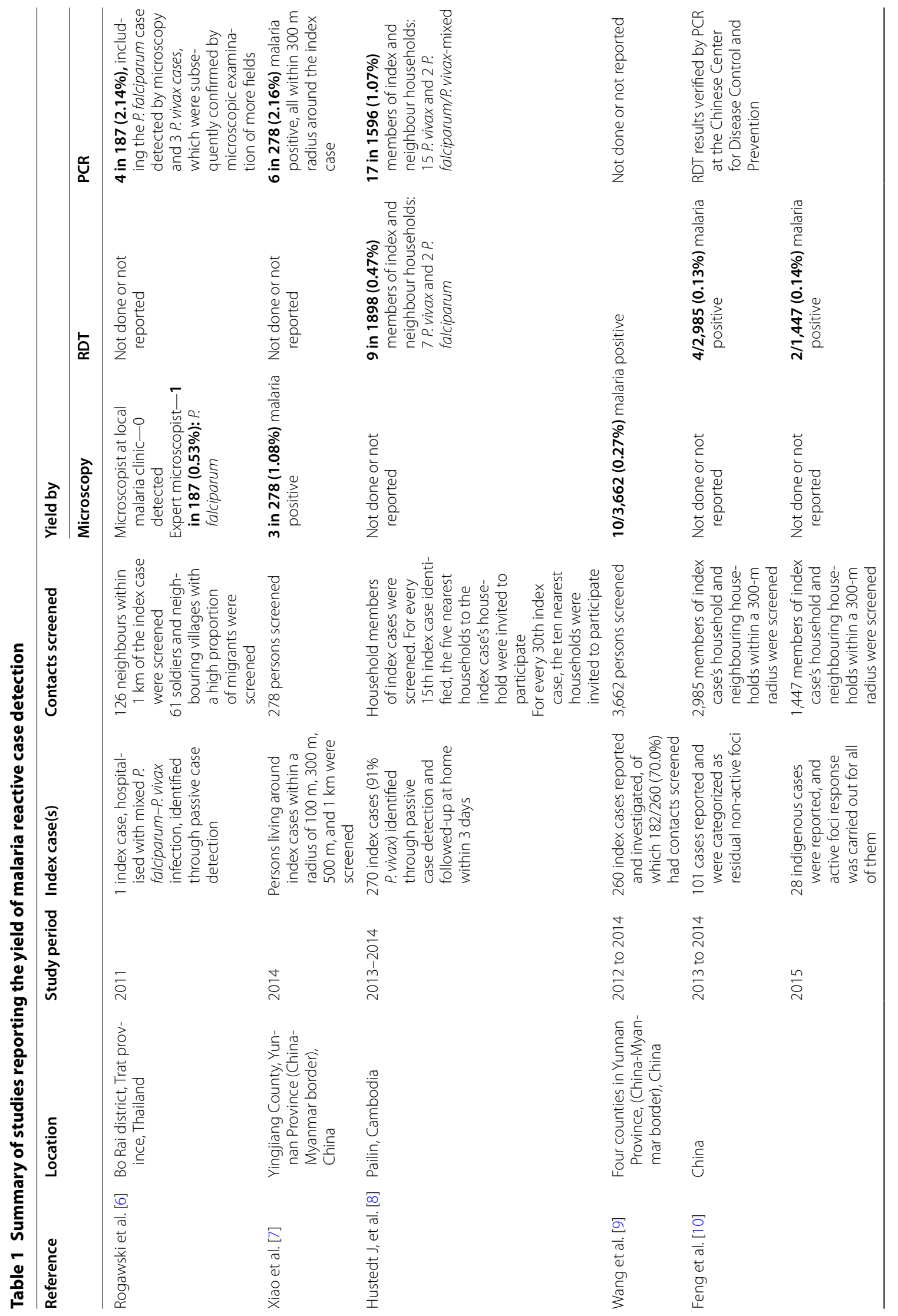




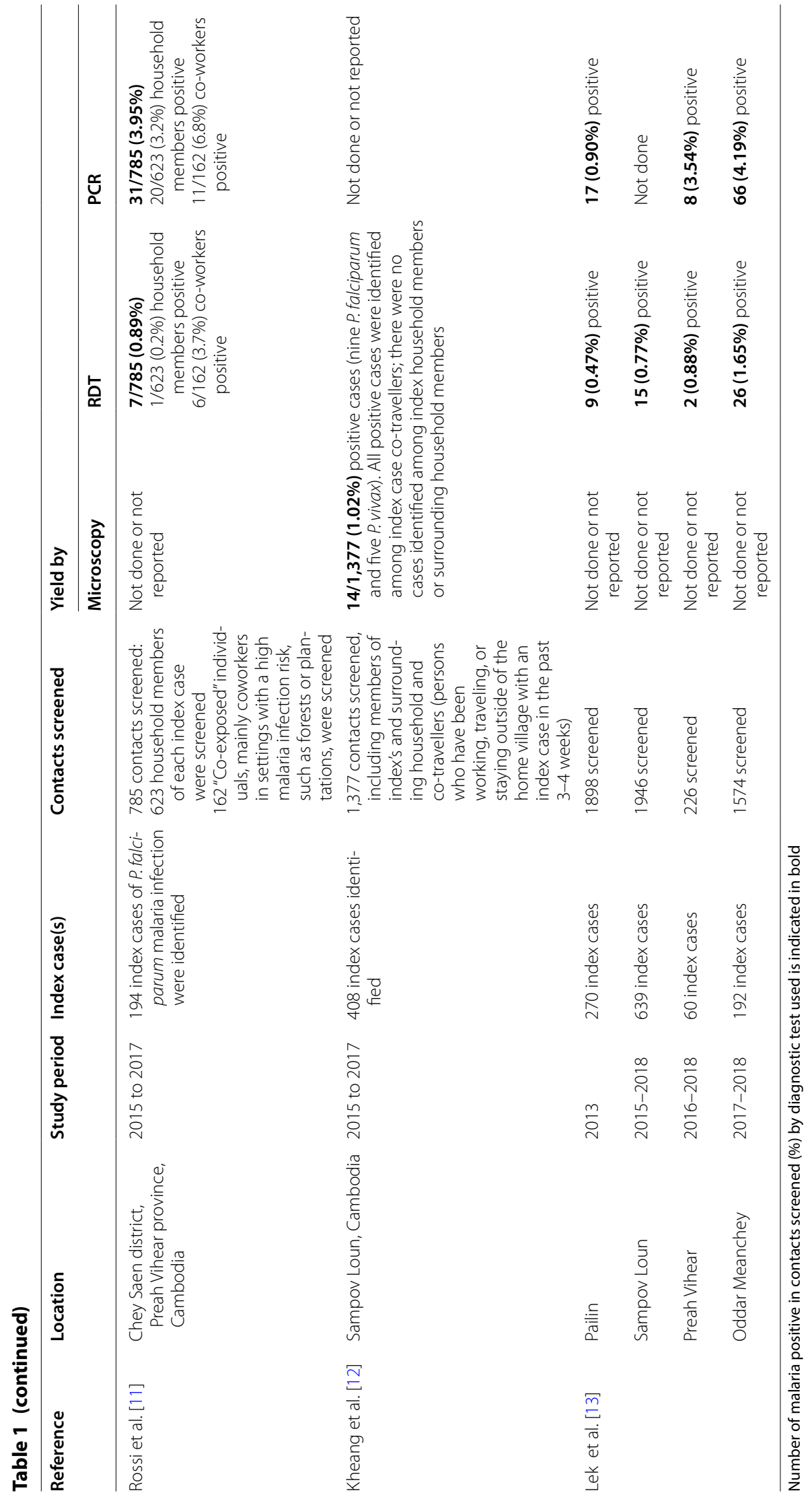




\section{a}

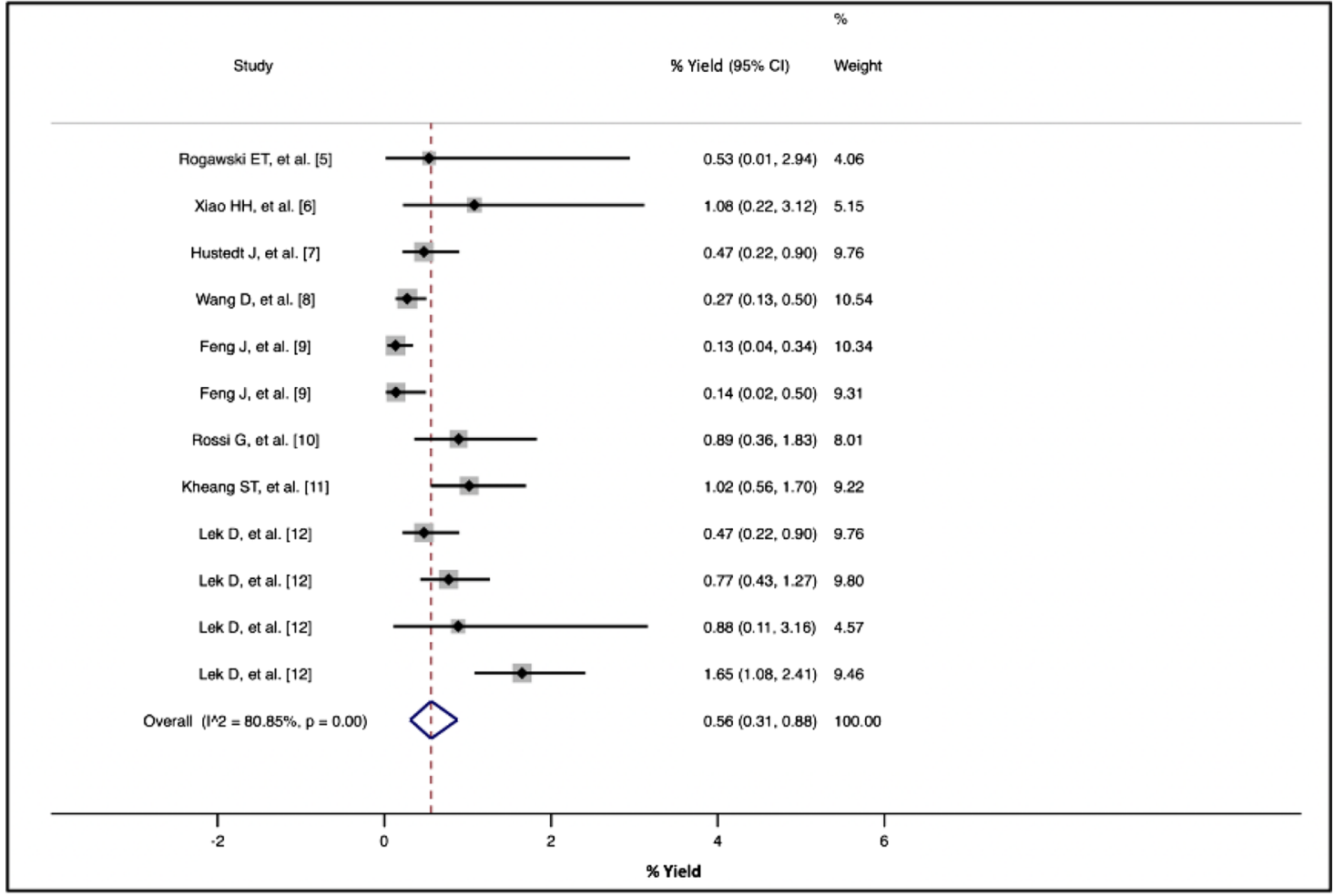

b

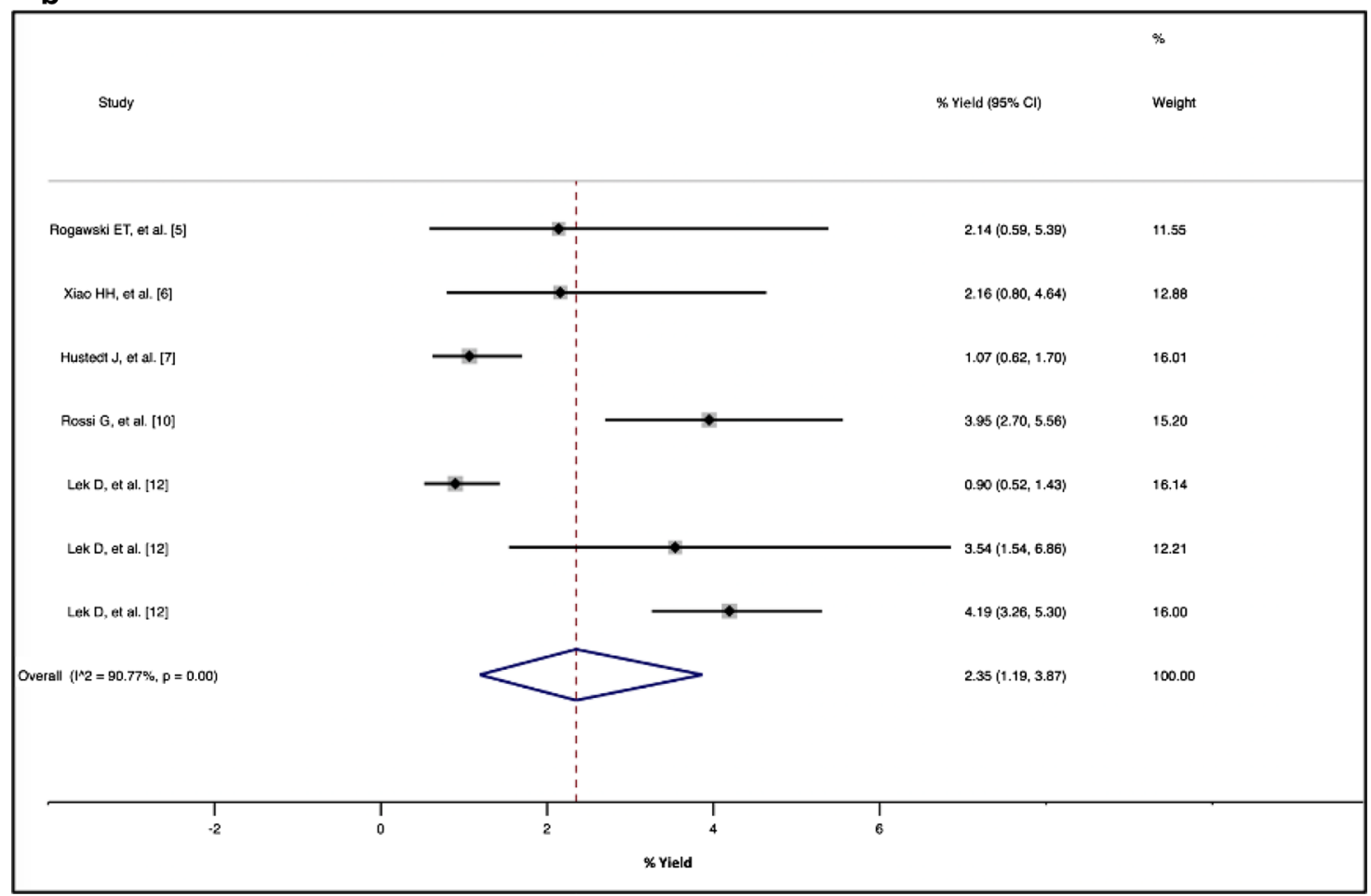

Fig. 2 a, b Metanalysis of reactive case detection (RACD) from published studies in the Greater Mekong Sub-region, by diagnostic test. a Percentage yield from RACD using microscopy and/or RDT for screening. b Percentage yield from RACD using PCR for screening 
miss about half of malaria positives, particularly low density infections [14]. Hopes that "highly sensitive" RDTs could solve these diagnostic challenges turned out to be misplaced. [13] "Highly sensitive" in context of RDTs is rather a misnomer because the additional yield compared to standard RDTs is minimal but the price is roughly twice that of standard RDTs.

Further restricting the yield of the RACD reported here is the primary focus on household contacts, an approach which was found to be effective in China where malaria transmission occurs within the household [3]. This approach may be less appropriate in the GMS where the large majority of infections occurs outdoors, in farms and forested areas. The people at risk are, therefore, those who work in the same location as the index case and not necessarily share the same household [15]. Only two studies in this review compared target groups and found higher yields from screening co-workers and co-travellers than household contacts $[11,12]$.

This review has several limitations. There were only a few published studies reporting the yield of RACD. Secondly, the studies were done in only some areas of the GMS. The majority of data for this review come from Cambodia with some additional data from China and Thailand. A more complete picture would require the inclusion of more data from Laos and Vietnam. Thirdly, the studies incorporated a wide variety of public health and research methodologies in the tracing and confirmation of contacts, which may question the value of aggregating these different datasets. In the absence of other published sources of information, this is the currently best available method to describe the yield of RACD in the GMS. Assessments of routinely collected data from the GMS malaria control programmes would be important to determine the actual yield of RACD. This review highlights the need for more standardized protocols in RACD, so that results can be compared by location and over time.

The implications for public health are multi-fold. If investigators have to test 200 people to detect a single case, their enthusiasm for RACD is likely to wane quickly. Perhaps it is more promising under the given circumstances to treat contacts presumptively with appropriate schizontocidal anti-malarials, i.e. not including the use of 8-aminoquinolines for radical cure of vivax malaria. Presumptive treatment avoids not only the costs for diagnostic tests which can be more expensive than a course of anti-malarials, but also the risk of missing cases due to the inadequate sensitivity of tests. Such presumptive treatment should probably consist of a full curative regimen using a drug combination to be determined in discussion with each National Malaria Control Program. The disadvantages of presumptive treatment is the reluctance of many contacts to receive treatment in the absence of testing and the increase in anti-malarial consumption leading to an increase in drug pressure. Alternatively, locally-appropriate evidence-based targeting of RACD, such as including household members in villages close to the forest but focusing on occupational contacts who share exposure in time and space with the index case in other areas.

Results from screening of contacts may be needed by national programmes to track progress towards malaria elimination and to support certification of elimination in the longer term. Presumptive treatment of contacts could still be carried-out but with the prior collection of dried blood on filter paper, labelled with the date and location. The dried blood smears could be transported centrally and batched-tested by PCR. The deferred PCR results would be used for identifying areas of continued transmission over time. Collection and PCR testing of dried blood smears will only be feasible in countries with available logistics and funds.

\section{Conclusion}

RACD has been adopted by malaria control programmes in the Greater Mekong Sub-region. Clearly the returns of RACD are diminishing as malaria transmission declines further and malaria elimination comes closer. Malaria control programmes may be faced with the question of whether the limited yield is worth the numerous resources required for RACD. Adapting RACD to presumptive treatment of contacts, with evidence-based targeting could increase the impact of this approach.

\section{Abbreviations}

PCD: Passive case detection; ACD: Active case detection; MMEV: Migrant Mobile Ethnic and Vulnerable populations; RACD: Reactive case detection; PACD: Proactive case detection; RDT: Rapid diagnostic test; PCR: Polymerase chain reaction; MDA: Mass drug administration.

\section{Acknowledgements \\ Not applicable}

\section{Authors' contributions}

All authors contributed to writing the manuscript. JD conceived the study and reviewed the articles. MM conducted the meta-analysis. JD and LvS interpreted the results and wrote the first draft. All authors read and approved the final manuscript.

\section{Funding}

None.

Availability of data and materials

All data generated or analysed during this review are included in this article.

\section{Declarations}

Ethics approval and consent to participate Not applicable. 


\section{Consent for publication}

Received from all co-authors.

\section{Competing interests}

The authors declare that they have no competing interests.

\section{Author details}

${ }^{1}$ Institute of Child Health and Human Development, National Institutes of Health, University of the Philippines, 623 P. Gil St, 1000 Manila, Philippines. ${ }^{2}$ Faculty of Tropical Medicine, Mahidol-Oxford Tropical Medicine Research Unit, Mahidol University, Bangkok, Thailand. ${ }^{3}$ Centre for Tropical Medicine and Global Health, Nuffield Department of Medicine, University of Oxford, Oxford, UK.

Received: 11 October 2020 Accepted: 24 February 2021

Published online: 04 March 2021

\section{References}

1. Sturrock HJ, Hsiang MS, Cohen JM, Smith DL, Greenhouse B, Bousema $\mathrm{T}$, et al. Targeting asymptomatic malaria infections: active surveillance in control and elimination. PLoS Med. 2013;10:e1001467.

2. WHO SEARO. Mobile and migrant populations and malaria information systems. New Delhi, World Health Organization, Regional Office for South-East Asia, 2015. https://apps.who.int/iris/bitstream/handle/10665 /204343/SEA-MAL-279.pdf;jsessionid=B89749B15B3AF36D0921C95AB 3C7B5BD? sequence $=1$ Accessed on 3 October 2020.

3. Cao J, Sturrock HJ, Cotter C, Zhou S, Zhou H, Liu Y, et al. Communicating and monitoring surveillance and response activities for malaria elimination: China's"1-3-7" strategy. PLoS Med. 2014;11:e1001642.

4. Parker DM, Landier J, von Seidlein L, Dondorp A, White L, Hanboonkunupakarn $B$, et al. Limitations of malaria reactive case detection in an area of low and unstable transmission on the Myanmar-Thailand border. Malar J. 2016;15:571.

5. Nyaga VN, Arbyn M, Aerts M. Metaprop: a Stata command to perform meta-analysis of binomial data. Arch Public Health. 2014;72:39.

6. Rogawski ET, Congpuong K, Sudathip P, Satimai W, Sug-aram R, Aruncharus $S$, et al. Active case detection with pooled real-time PCR to eliminate malaria in Trat province. Thailand Am J Trop Med Hyg. 2012;86:789-91.

7. Xiao HH, Liu J, Feng J, Zhang SS, Jiang WK, Xia ZG, et al. [Screening radius of active case detection and the malaria parasite rate of carriers in China-Myanmar Border](in Chinese). Zhongguo Ji Sheng Chong Xue Yu Ji Sheng Chong Bing Za Zhi. 2015;33:86-90.

8. Hustedt J, Canavati SE, Rang C, Ashton RA, Khim N, Berne L, et al. Reactive case-detection of malaria in Pailin Province, Western Cambodia: lessons from a year-long evaluation in a pre-elimination setting. Malar J. 2016;15:132.

9. Wang D, Cotter C, Sun X, Bennett A, Gosling RD, Xiao N. Adapting the local response for malaria elimination through evaluation of the 1-3-7 system performance in the China-Myanmar border region. Malar J. 2017;16:54.

10. Feng J, Tu H, Zhang L, Zhang S, Jiang S, Xia Z, et al. Mapping transmission foci to eliminate malaria in the People's Republic of China, 2010-2015: a retrospective analysis. BMC Infect Dis. 2018;18:115.

11. Rossi G, Van den Bergh R, Nguon C, Debackere M, Vernaeve L, Khim N, et al. Adapting reactive case detection strategies for falciparum malaria in a low-transmission area in Cambodia. Clin Infect Dis. 2018;66:296-8.

12. Kheang ST, Sovannaroth S, Barat LM, Dysoley L, Kapella BK, Po L, et al. Malaria elimination using the 1-3-7 approach: lessons from Sampov Loun. Cambodia BMC Public Health. 2020;20:544.

13. Lek D, Callery JJ, Nguon C, Debackere M, Sovannaroth S, Tripura R, et al. Tools to accelerate falciparum malaria elimination in Cambodia: a meeting report. Malar J. 2020;19:151.

14. Imwong M, Stepniewska K, Tripura R, Peto TJ, Lwin KM, Vihokhern B, et al. Numerical distributions of parasite densities during asymptomatic malaria. J Infect Dis. 2016;213:1322-9.

15. von Seidlein L, Peto TJ, Tripura R, Pell C, Yeung S, Kindermans JM, et al. Novel approaches to control malaria in forested areas of Southeast Asia. Trends Parasitol. 2019;35:388-98.

\section{Publisher's Note}

Springer Nature remains neutral with regard to jurisdictional claims in published maps and institutional affiliations.
Ready to submit your research? Choose BMC and benefit from:

- fast, convenient online submission

- thorough peer review by experienced researchers in your field

- rapid publication on acceptance

- support for research data, including large and complex data types

- gold Open Access which fosters wider collaboration and increased citations

- maximum visibility for your research: over $100 \mathrm{M}$ website views per year

At BMC, research is always in progress.

Learn more biomedcentral.com/submissions 